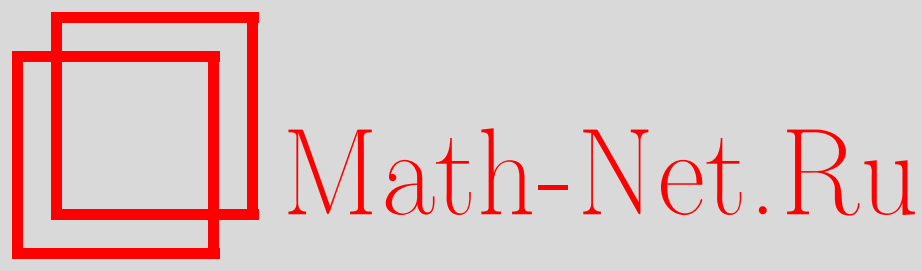

М. Л. Гандариас, Нелокальные симметрии и редукции для некоторых обыкновенных дифференциальных уравнений, ТМФ, 2009, том 159, номер 3, 428-437

DOI: https://doi.org/10.4213/tmf6362

Использование Общероссийского математического портала Math-Net.Ru подразумевает, что вы прочитали и согласны с пользовательским соглашением http://www.mathnet.ru/rus/agreement

Параметры загрузки:

IP : 54.198.55.26

26 апреля 2023 г., 05:25:26

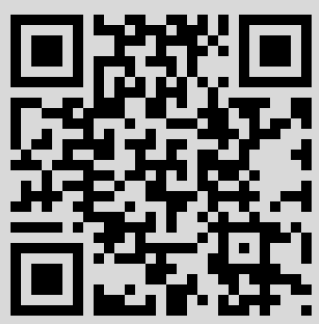




\title{
ФИЗИКА
}

Том 159, № 3

июнь, 2009

\section{НЕЛОКАЛЬНЫЕ СИММЕТРИИ И РЕДУКЦИИ ДЛЯ НЕКОТОРЫХ ОБЫКНОВЕННЫХ ДИФФЕРЕНЦИАЛЬНЫХ УРАВНЕНИЙ}

\begin{abstract}
Приведен вывод нелокальных симметрий для обыкновенных дифференциальных уравнений. Эти симметрии найдены путем помещения уравнения во вспомогательную систему. С использованием нелокальных симметрий показано, что порядок обыкновенного дифференциального уравнения можно понизить даже в случае, когда оно не допускает локальных симметрий.
\end{abstract}

Ключевые слова: условная симметрия, нелокальная симметрия, обыкновенное дифференциальное уравнение.

\section{1. ВВЕДЕНИЕ}

Хорошо известно, что если обыкновенное дифференциальное уравнение (ОДУ) обладает симметрией, задаваемой однопараметрической группой Ли, то порядок этого уравнения можно уменьшить на единицу. В частности, ОДУ первого порядка, для которого известна однопараметрическая группа симметрий, может быть проинтегрировано с помощью единственной квадратуры. В случае групп симметрий старших порядков ситуация становится несколько иной, и, вообще говоря, не представляется возможным уменьшить на $r$ порядок уравнения, инвариантного относительно действия $r$-параметрической группы симметрий, используя только квадратуры: необходимо, чтобы сама группа была разрешимой. Таким образом, одного только наличия симметрий не всегда достаточно для интегрируемости дифференциального уравнения в квадратурах. Обратное утверждение, а именно то, что каждое дифференциальное уравнение, интегрируемое в квадратурах, обладает нетривиальной группой симметрий, как оказалось, не имеет места. Это было доказано в работе [1], в которой приведен пример ОДУ второго порядка, интегрируемого в квадратурах, но обладающего только тривиальной группой симметрий.

В последние несколько лет наблюдался значительный прогресс в применении симметрий к исследованиям редукций ОДУ. Очевидно, что использование теоретико-групповых методов, основанных на локальных симметриях, ограничивается

* Departamento de Matemáticas, Universidad de Cádiz, PO.BOX 40, 11510 Puerto Real, Cádiz, Spain. E-mail: marialuz.gandarias@uca.es 
тем, что многие ОДУ не обладают локальными симметриями. Оказывается тем не менее, что ОДУ могут обладать нелокальными симметриями, инфинитезимальные генераторы которых зависят от интегралов от зависимых переменных некоторым специальным образом.

Нелокальные симметрии были введены в работах [2]-[5]. Потенциальные симметрии, которые представляют собой специальный случай общих нелокальных симметрий, были введены для ОДУ в работе [6]. Пусть

$$
\Delta\left(x, u, \frac{d u}{d x}, \ldots, \frac{d^{n} u}{d x^{n}}\right)=0
$$

- ОДУ $n$-го порядка $(n \geqslant 2)$, где $\Delta(\cdot)$ - некоторая функция от указанных аргументов. Предположим, что существует задающее вспомогательную переменную $v$ преобразование $u=f\left(x, v, v^{(1)}\right)$ (здесь и далее $f(\cdot)$ обозначает некоторую функцию) такое, что уравнение (1) можно переписать в виде закона сохранения

$$
\frac{D}{D x} \widetilde{\Delta}\left(x, v, \frac{d v}{d x}, \ldots, \frac{d^{n-1} v}{d x^{n-1}}\right)=0
$$

при некоторой функции $\widetilde{\Delta}(\cdot)$ от указанных аргументов. Здесь

$$
\frac{D}{D x}=\frac{\partial}{\partial x}+v_{x} \frac{\partial}{\partial v}+v_{x x} \frac{\partial}{\partial v_{x}}+\cdots
$$

- оператор полной производной. Применяя закон сохранения (2), можно образовать вспомогательную потенциальную систему

$$
v_{x}=f(x, u), \quad \widetilde{\Delta}\left(x, v, \frac{d v}{d x}, \ldots, \frac{d^{n-1} v}{d x^{n-1}}\right)=0 .
$$

В данном случае оператор полной производной задается выражением

$$
\frac{D}{D x}=\frac{\partial}{\partial x}+u_{x} \frac{\partial}{\partial u}+v_{x} \frac{\partial}{\partial v}+u_{x x} \frac{\partial}{\partial u_{x}}+v_{x x} \frac{\partial}{\partial v_{x}}+\cdots .
$$

Любая группа Ли локальных преобразований вида

$$
\mathbf{v}=\xi(x, u, v) \frac{\partial}{\partial x}+\phi(x, u, v) \frac{\partial}{\partial u}+\psi(x, u, v) \frac{\partial}{\partial v},
$$

допускаемых (4), задает нелокальную потенииальную симметрию ОДУ (2), если выполнено следующее условие:

$$
\xi_{v}^{2}+\phi_{v}^{2} \neq 0
$$

Потенциальные симметрии для некоторых ОДУ были построены в работах [7], [8]. Применяя эти симметрии, можно понизить порядок ОДУ даже в том случае, когда уравнение не допускает локальных симметрий. Более того, в случае, когда ОДУ допускает группу локальных симметрий, оказывается, что потенциальные симметрии позволяют произвести более основательные редукции по сравнению с редукциями, связанными с локальными симметриями. 
Конструктивный метод построения нелокальных симметрий дифференциальных уравнений, основанный на теории групп Ли-Беклунда, был развит в работе [9]. В монографии [10] были введены экспоненциированные векторные поля, а также было доказано, что степень ОДУ, инвариантного относительно действия экспоненциированного векторного поля, может быть понижена на единицу. В работе [11] были введены $\lambda$-симметрии, также понижающие порядок ОДУ. Соотношение между ними и нелокальными симметриями было найдено в работах [12], [13]. Нелокальные симметрии применялись при интегрировании или при уменьшении порядка ОДУ в работах [14]-[19]. Как правило, эти нелокальные симметрии проявляются как скрытые симметрии ОДУ, получаемые при преобразовании $u=f\left(x, v_{x}\right)$, которое увеличивает порядок уравнения. Основной трудностью при этом оказывается отсутствие регулярных методов построения таких уравнений старших порядков. Более того, вычислительные программы типа symmgrp.max не способны находить симметрии Ли ОДУ, в котором было произведено преобразование $u=f\left(x, v_{x}\right)$, так что сначала необходимо угадать возможный вид функции $f$ и лишь затем вычислить симметрии.

В настоящей работе мы предлагаем находить полезные нелокальные симметрии ОДУ с помощью погружения этого уравнения во вспомогательную "обертывающую" систему с вспомогательными зависимыми переменными. Симметрия Ли вспомогательной системы, действующая на пространстве, содержащем независимые и зависимые переменные данного ОДУ, равно как и вспомогательные переменные, задает нелокальную симметрию для исходного ОДУ, если она не проецируется в локальную симметрию, действующую на пространстве независимых и зависимых переменных. Введем вспомогательную нелокальную переменную $v$ и вспомогательную систему

$$
\Delta\left(x, u, \frac{d u}{d x}, \ldots, \frac{d^{n} u}{d x^{n}}\right)=0, \quad v_{x}=f(x, u) .
$$

Любая группа Ли точечных преобразований вида (6), разрешенных системой (8), задает нелокальную симметрию исходного ОДУ (2), если выполнено условие (7). Нелокальные симметрии уравнения (2) представляют собой локальные симметрии системы (8), таким образом мы приходим к алгоритму вывода подобных нелокальных симметрий. Это позволяет использовать компьютер для обычно весьма трудоемких аналитических вычислений.

Мы укажем несколько классов ОДУ, которые не допускают никаких симметрий Ли, но для которых можно получить нелокальные симметрии, и это позволяет уменьшить порядок исходных уравнений.

\section{2. НЕЛОКАЛЬНЫЕ СИММЕТРИИ}

2.1. Рассмотрим ОДУ второго порядка

$$
u_{x x}=\frac{\left(u_{x}\right)^{2}}{u}+\left(c(x) u^{n}+b(x)\right) u_{x}+\left(c^{\prime}(x)-c(x) b(x)\right) \frac{u^{n+1}}{n}+d(x) u
$$

как пример уравнения, которое не допускает локальные симметрии Ли, если только $b(x), c(x)$ и $d(x)$ не удовлетворяют некоторым условиям. Следовательно, это уравнение не может быть в общем случае проинтегрировано с помощью метода симметрий 
Ли. Мы докажем, что связанная с этим уравнением вспомогательная система тем не менее допускает симметрии Ли, которые проявляются как нелокальные симметрии уравнения (9). С их помощью можно понизить порядок этого уравнения и проинтегрировать его в квадратурах.

В работе [1] было предложено рассматривать

$$
u_{x x}=\frac{\left(u_{x}\right)^{2}}{u}+n c(x) u^{n} u_{x}+c^{\prime}(x) u^{n+1}
$$

в качестве примера уравнения, которое интегрируется в квадратурах и не имеет нетривиальных симметрий Ли, если только $c(x)$ не является одной из следующих функций:

$$
c(x)=k_{1} e^{k_{2} x}\left(k_{3}+k_{4} x\right)^{k_{5}}, \quad c(x)=e^{k_{6} x^{2}} .
$$

Тем самым для любой другой функции $c(x)$ это уравнение не может быть проинтегрировано с помощью метода симметрий Ли. Уравнение (10) представляет собой частный случай уравнения (9), в котором выбрано $b(x)=0$ и $d(x)=0$.

ТЕОРема 2.1. ОДУ второго порядка (9) допускает симметрии Ли с генераторами инфинитезимальных преобразований

$$
\xi=\xi(x), \quad \phi=-\frac{1}{n c}(c \xi)^{\prime} u
$$

тогда и только тогда, когда выполнены следующие условия:

$$
\begin{gathered}
\xi^{\prime \prime}+(b \xi)^{\prime}=0 \\
-\frac{2}{n c^{2}}\left(c^{2} d n+c c^{\prime \prime}-\left(c^{\prime}\right)^{2}-b c c^{\prime}-b^{\prime} c^{2}+b^{2} c^{2}\right)\left(k_{1}-b \xi\right)=0 .
\end{gathered}
$$

ДоказАтЕльство. Если векторное поле $\mathbf{u}=\xi \partial / \partial x+\phi \partial / \partial u$ представляет собой симметрию Ли уравнения (9), то его генераторы $\xi$, $\phi$ должны удовлетворять следующим определяющим уравнениям:

$$
\begin{gathered}
\frac{\partial^{2} \xi}{\partial u^{2}} u+\frac{\partial \xi}{\partial u}=0 \\
\frac{\partial^{2} \phi}{\partial u^{2}} u^{2}-2 \frac{\partial \xi}{\partial u} f u^{2}-2 \frac{\partial^{2} \xi}{\partial u \partial x} u^{2}-\frac{\partial \phi}{\partial u} u+\phi=0 \\
\frac{\partial^{2} \phi}{\partial x^{2}}-f \frac{\partial \phi}{\partial x}+g \frac{\partial \phi}{\partial u}-\frac{\partial g}{\partial u} \phi-\xi \frac{\partial g}{\partial x}-2 \frac{\partial \xi}{\partial x} g=0 \\
-2 \frac{\partial^{2} \phi}{\partial u \partial x} u+\frac{\partial f}{\partial u} \phi u+3 \frac{\partial \xi}{\partial u} g u+\xi \frac{\partial f}{\partial x} u+\frac{\partial \xi}{\partial x} f u+\frac{\partial^{2} \xi}{\partial x^{2}} u+2 \frac{\partial \phi}{\partial x}=0,
\end{gathered}
$$

где $f(x, u)=c(x) u^{n}+b(x)$ и $g(x, u)=\left(c^{\prime}(x)-c(x) b(x)\right) u^{n+1} / n+d(x) u$. Решая первое из определяющих уравнений, получим $\xi=f_{1} \ln u+f_{2}$, подставляя это выражение во второе уравнение, имеем

$$
\phi=\frac{\left(f_{1}^{\prime}+b f_{1}\right) u \ln ^{2} u^{n}+2 c f_{1} u^{n+1}}{n^{2}}+u\left(f_{3} \ln u+f_{4}\right)
$$

с $f_{i}=f_{i}(x)$ для $i=1, \ldots, 4$. Подставим $\xi$ и $\phi$ в оставшиеся уравнения, приравняем коэффициенты при $u^{2 n}, \ln u$ и $u^{n}$ к нулю, в результате получим, что $f_{1}=f_{3}=0$, 
$f_{4}=\left(c f_{2}\right)^{\prime} / n c$, a $f_{2}, b, c$ и $d$ должны удовлетворять системе

$$
\begin{gathered}
f_{2}^{\prime \prime}+\left(b f_{2}\right)^{\prime}=0 \\
-\frac{2}{n c^{2}}\left(c^{2} d n+c c^{\prime \prime}-\left(c^{\prime}\right)^{2}-b c c^{\prime}-b^{\prime} c^{2}+b^{2} c^{2}\right)\left(k_{1}-b f_{2}\right)=0 .
\end{gathered}
$$

Теорема доказана.

Так, при $c(x)=x, b(x)=1, d(x)=1$ уравнение (9) не имеет симметрий Ли.

Вводя нелокальную переменную $v$, можно сформулировать следующую теорему.

TEOPEMA 2.2. Cuсmeмa

$$
\begin{gathered}
u_{x x}=\frac{\left(u_{x}\right)^{2}}{u}+\left(c(x) u^{n}+b(x)\right) u_{x}+\left(c^{\prime}(x)-c(x) b(x)\right) \frac{u^{n+1}}{n}+d(x) u, \\
v_{x}=h(x, u)
\end{gathered}
$$

имеет симметрии Ли с инфинитезимальным генератором

$$
\mathbf{v}=a(x) e^{v} u \frac{\partial}{\partial u}+n a(x) k e^{v} \frac{\partial}{\partial v}
$$

$\operatorname{ecлu~} h(x, u)=c(x) u^{n}-a^{\prime}(x) / a(x)$.

ДокАзАтЕЛьСтво. Если векторное поле

$$
\mathbf{v}=\xi \frac{\partial}{\partial x}+\phi \frac{\partial}{\partial u}+\psi \frac{\partial}{\partial v}
$$

представляет собой симметрию Ли уравнения (16), то его генераторы $\xi, \phi$ и $\psi$ должны удовлетворять следующим определяющим уравнениям:

$$
\begin{gathered}
\frac{\partial^{2} \xi}{\partial u^{2}} u+\frac{\partial \xi}{\partial u}=0, \quad \frac{\partial \psi}{\partial u}-\frac{\partial \xi}{\partial u} h=0 \\
\frac{\partial \psi}{\partial x}+h \frac{\partial \psi}{\partial v}-\frac{\partial h}{\partial u} \phi-\xi \frac{\partial h}{\partial x}-\frac{\partial \xi}{\partial v} h^{2}-\frac{\partial \xi}{\partial x} h=0 \\
\frac{\partial^{2} \phi}{\partial u^{2}} u^{2}-\frac{\partial \xi}{\partial v} \frac{\partial h}{\partial u} u^{2}-2 \frac{\partial^{2} \xi}{\partial u \partial v} h u^{2}-2 \frac{\partial \xi}{\partial u} f u^{2}-2 \frac{\partial^{2} \xi}{\partial u \partial x} u^{2}-\frac{\partial \phi}{\partial u} u+\phi=0 \\
\frac{\partial^{2} \phi}{\partial x^{2}}-f \frac{\partial \phi}{\partial x}+h^{2} \frac{\partial^{2} \phi}{\partial v^{2}}+2 h \frac{\partial^{2} \phi}{\partial v \partial x}+\frac{\partial h}{\partial x} \frac{\partial \phi}{\partial v}-f h \frac{\partial \phi}{\partial v}+ \\
+g \frac{\partial \phi}{\partial u}-\frac{\partial g}{\partial u} \phi-2 \frac{\partial \xi}{\partial v} g h-\xi \frac{\partial g}{\partial x}-2 \frac{\partial \xi}{\partial x} g=0 \\
\frac{\partial h}{\partial u} \frac{\partial \phi}{\partial v} u+2 \frac{\partial^{2} \phi}{\partial u \partial x} u+2 h \frac{\partial^{2} \phi}{\partial u \partial v} u \frac{\partial f}{\partial u} \phi u- \\
-\frac{\partial \xi}{\partial v} \frac{\partial h}{\partial x} u-\frac{\partial^{2} \xi}{\partial v^{2}} h^{2} u-\frac{\partial \xi}{\partial v} f h u-2 \frac{\partial^{2} \xi}{\partial v} h u-3 \frac{\partial \xi}{\partial u} g u- \\
-\xi \frac{\partial f}{\partial x} u-\frac{\partial \xi}{\partial x} f u-\frac{\partial^{2} \xi}{\partial x^{2}} u-2 \frac{\partial \phi}{\partial x}-2 h \frac{\partial \phi}{\partial v}=0
\end{gathered}
$$

где $f(x, u)=c(x) u^{n}+b(x)$ и $g(x, u)=\left(c^{\prime}(x)-c(x) b(x)\right) u^{n+1} / n+d(x) u$. 
Можно проверить, что эта система допускает решение $\xi=0, \phi=a(x) e^{v} u, \psi=$ $n a(x) k e^{v}$ и $h(x, u)=c(x) u^{n}-a^{\prime}(x) / a(x)$. Следовательно, она обладает симметриями Ли с генератором (17). Теорема доказана.

С использованием формулы продолжения получим, что

$$
\mathbf{v}^{(1)}=a(x) e^{v} u \frac{\partial}{\partial u}+a(x) e^{v} \frac{\partial}{\partial v}+e^{v}\left(a(x) u_{x}+a(x) c(x) u^{n+1}\right) \frac{\partial}{\partial u_{x}},
$$

при этом двумя функционально независимыми инвариантами будут

$$
z=x, \quad \zeta=\frac{u_{x}}{u}-\frac{c(x) u^{n}}{n} .
$$

Взяв производную, мы получаем, что уравнение (9), записанное в терминах инвариантов, оказывается линейным уравнением первого порядка $\zeta_{z}-b(z) \zeta-d(z)=0$, общее решение которого имеет вид

$$
\zeta=e^{\int b(z) d x}\left(\int d(z) e^{-\int b(z) d x} d x+k_{1}\right) .
$$

Уравнение

$$
\frac{u_{z}}{u}-\frac{c(z) u^{n}}{n}=\zeta(z)
$$

дает

$$
u=\frac{e^{\int \zeta(z) d z}}{\left(k_{2}-c \int e^{n \int \zeta(z) d z} d z\right)^{1 / n}},
$$

где $\zeta$ задается формулой (21).

Дифференциальное уравнение второго порядка

$$
u_{x x}-\frac{\left(u_{x}\right)^{2}}{u}-\frac{x^{2}+x}{u} u_{x}+2 x+1=0
$$

было недавно предложено в работе [20] в качестве примера уравнения, не имеющего локальных симметрий и, следовательно, не интегрируемого с помощью метода симметрий Ли. Для уравнений старших порядков, приведенных в работе [20], был применен метод интегрирующих множителей, и решение выражено в терминах элементарных функциях. Уравнение (22) представляет собой частный случай уравнения (9), в котором $c(x)=x^{2}+x, n=-1$ и $b(x)=d(x)=0$. Соответственно получим, что, хотя уравнение (22) не имеет точечных симметрий, введение нелокальной переменной $v$ приводит к тому, что связанная с ней система допускает симметрии Ли, которые оказываются нелокальными симметриями уравнения (22). Следующая теорема является частным случаем теоремы 2.1.

TeOpema 2.3. Система

$$
u_{x x}-\frac{\left(u_{x}\right)^{2}}{u}-\frac{x^{2}+x}{u} u_{x}+2 x+1=0, \quad v_{x}=f(x, u)
$$

имеет симметрии Ли с инфинитезимальным генератором

$$
\mathbf{v}=e^{v} u \frac{\partial}{\partial u}+\left(k_{1}-e^{v}\right) \frac{\partial}{\partial v},
$$

если $f(x, u)=\left(x^{2}+x\right) / u$. 
Применим обычную формулу продолжения, тогда

$$
\mathbf{v}^{(1)}=e^{v} u \frac{\partial}{\partial u}+\left(k_{1}-e^{v}\right) \frac{\partial}{\partial v}+e^{v} u_{x} \frac{\partial}{\partial u_{x}}+e^{v} u v_{x} \frac{\partial}{\partial v_{x}} .
$$

Два функционально независимых инварианта имеют вид

$$
z=x, \quad \zeta=\frac{u_{x}}{u}+\frac{x^{2}+x}{u} .
$$

Взяв производную, получим, что уравнение (22) в терминах инвариантов задается тривиальным уравнением первого порядка $\zeta_{z}=0$, имеющим $\zeta=C$ в качестве общего решения. В терминах исходных переменных последнее равенство записывается как

$$
\frac{u_{x}+x^{2}+x}{u}=C .
$$

2.2. Далее мы будем рассматривать ОДУ второго порядка

$$
u_{x x}-d(x) u+\frac{k c_{x}(x)}{2 u}+\frac{k^{2} c^{2}(x)}{4 u^{3}}=0,
$$

которое не обладает симметриями Ли, если только функции $c(x)$ и $d(x)$ не удовлетворяют некоторым специальным условиям.

ТЕОРема 2.4. ОДУ второго порядка (27) не имеет нетривиалъных симметрий Ли, если только функиии $c(x)$ и $d(x)$ не удовлетворяют следующему условию:

$$
\left(\frac{c}{c_{x}}\right)_{x x x}-4 d\left(\frac{c}{c_{x}}\right)_{x}-2 d_{x}\left(\frac{c}{c_{x}}\right)=0
$$

$u$, следовательно, для любых функиий $c(x)$ u $d(x)$, не удовлетворяющих (28), уравнение (27) не может быть проинтегрировано методом симметрий Ли.

ДокАзАтЕльство. Если векторное поле $\mathbf{v}=\xi \partial / \partial x+\phi \partial / \partial u$ представляет собой симметрию Ли уравнения (27), то его генераторы $\xi$ и $\phi$ должны удовлетворять следующим определяющим уравнениям:

$$
\begin{gathered}
\frac{\partial^{2} \xi}{\partial u^{2}}=0, \quad \frac{\partial^{2} \phi}{\partial u^{2}}-2 \frac{\partial^{2} \xi}{\partial u \partial x}=0, \\
2 \frac{\partial^{2} \phi}{\partial u \partial x}-3 \frac{\partial \xi}{\partial u} f-\frac{\partial^{2} \xi}{\partial x^{2}}=0, \\
\frac{\partial^{2} \phi}{\partial x^{2}}+f \frac{\partial \phi}{\partial u}-\frac{\partial f}{\partial u} \phi-\xi \frac{\partial f}{\partial x}-2 \frac{\partial \xi}{\partial x} f=0,
\end{gathered}
$$

где

$$
f=d(x) u-\frac{k c_{x}(x)}{2 u}-\frac{k^{2} c^{2}(x)}{4 u^{3}} .
$$

Решая первое из определяющих уравнений, получим $\xi=f_{1}(x) u+f_{2}(x)$ и, подставив это решение во второе уравнение, имеем $\phi=f_{1}^{\prime}(x) u^{2}+f_{2}(x) u+f_{3}(x)$. Подставив эти выражения в оставшиеся уравнения и положив постоянные члены равными нулю, получим, что $f_{1}=f_{4}=0$ и $f_{3}=\left(f_{2}\right)^{\prime} / 2+k_{1}$, а $c(x)$ и $d(x)$ должны удовлетворять условию (28). Теорема доказана. 
Тем не менее справедлива следующая

TEOPEMA 2.5. Cucmema

$$
u_{x x}-d(x) u+\frac{k c_{x}(x)}{2 u}+\frac{k^{2} c^{2}(x)}{4 u^{3}}=0, \quad v_{x}=f(x, u)
$$

обладает симметриями Ли с инфинитезимальным генератором

$$
\mathbf{v}=a(x) e^{v} u \frac{\partial}{\partial u}-\frac{2 a(x)}{k} e^{v} \frac{\partial}{\partial v}
$$

если $f(x, u)=c(x) / u^{2}-a_{x} / k a$.

ДоказАтЕЛьСтво. Если векторное поле

$$
\mathbf{v}=\xi \frac{\partial}{\partial x}+\phi \frac{\partial}{\partial u}+\psi \frac{\partial}{\partial v}
$$

задает симметрию Ли системы (32), то его генераторы $\xi, \phi$ и $\psi$ должны удовлетворять следующей определяющей системе уравнений:

$$
\begin{gathered}
\frac{\partial^{2} \xi}{\partial u^{2}}=0, \quad \frac{\partial \psi}{\partial u}-\frac{\partial \xi}{\partial u} f=0 \\
\frac{\partial^{2} \phi}{\partial u^{2}}-\frac{\partial \xi}{\partial v} \frac{\partial f}{\partial u}-2 \frac{\partial^{2} \xi}{\partial u \partial v} f-2 \frac{\partial^{2} \xi}{\partial u \partial x}=0 \\
\frac{\partial \psi}{\partial x}+f \frac{\partial \psi}{\partial v}-\frac{\partial f}{\partial u} \phi-\xi \frac{\partial f}{\partial x}-\frac{\partial \xi}{\partial v} f^{2}-\frac{\partial \xi}{\partial x} f=0 \\
\frac{\partial f}{\partial u} \frac{\partial \phi}{\partial v}+2 \frac{\partial^{2} \phi}{\partial u \partial x}+2 f \frac{\partial^{2} \phi}{\partial u \partial v}-3 \frac{\partial \xi}{\partial u} g-\frac{\partial \xi}{\partial v} \frac{\partial f}{\partial x}-\frac{\partial^{2} \xi}{\partial v^{2}} f^{2}-2 \frac{\partial^{2} \xi}{\partial v \partial x} f-\frac{\partial^{2} \xi}{\partial x^{2}}=0 \\
\frac{\partial^{2} \phi}{\partial x^{2}}+f^{2} \frac{\partial^{2} \phi}{\partial v^{2}}+2 f \frac{\partial^{2} \phi}{\partial v \partial x}+\frac{\partial f}{\partial x} \frac{\partial \phi}{\partial v}+g \frac{\partial \phi}{\partial u}-\frac{\partial g}{\partial u} \phi-\xi \frac{\partial g}{\partial x}-2 \frac{\partial \xi}{\partial v} f g-2 \frac{\partial \xi}{\partial x} g=0
\end{gathered}
$$

Можно проверить, что система имеет решение $\xi=0, \phi=a(x) e^{v} u, \psi=-2 a(x) / k \cdot e^{v}$ и $f(x, u)=x^{n} / u^{2}-a_{x} / k a$. Следовательно, она имеет симметрии Ли с генератором (32). Теорема доказана.

Полагая, например, $c(x)=x^{n}$, получим, что уравнение

$$
u_{x x}-d(x) u+\frac{k n x^{n-1}}{2 u}+\frac{k^{2} x^{2 n}}{4 u^{3}}=0
$$

не допускает симметрий Ли при $d(x) \neq c_{1} / x^{2}$. Тем не менее система

$$
u_{x x}-d(x) u+\frac{k n x^{n-1}}{2 u}+\frac{k^{2} x^{2 n}}{4 u^{3}}=0, \quad v_{x}=f(x, u)
$$

при $f(x, u)=x^{n} / u^{2}-a_{x} / k a$ имеет симметрии Ли с инфинитезимальным генератором (32). Применяя формулу продолжения, получим

$$
\mathbf{v}^{(1)}=a(x) e^{k v} u \frac{\partial}{\partial u}-\frac{2 a(x)}{k} e^{k v} \frac{\partial}{\partial v}+e^{k v}\left(a(x) u_{x}+\frac{k a(x) x^{n}}{u}\right) \frac{\partial}{\partial u_{x}} .
$$


Два функционально независимых инварианта имеют вид

$$
z=x, \quad \zeta=\frac{u_{x}}{u}+\frac{k x^{n}}{2 u^{2}} .
$$

Взяв производную, получим, что уравнение (27) в терминах инвариантов представляет собой уравнение первого порядка $\zeta_{z}+\zeta^{2}-d(z)=0$. Положив $d(z)=1$, выпишем его общее решение $\zeta=\operatorname{th}\left(x+c_{1}\right)$ и соответствующее решение для $u$, заданное в неявном виде:

$$
4 k \int \frac{x^{n} e^{2\left(x+c_{1}\right)}}{\left(e^{2\left(x+c_{1}\right)}+1\right)^{2}} d x+\frac{u^{2}}{\operatorname{ch}^{2}\left(x+c_{1}\right)}-c_{2}=0 .
$$

Более того, можно проверить, что если векторное поле

$$
\mathbf{v}=a(x) e^{k v} u \frac{\partial}{\partial u}-\frac{2 a(x)}{k} e^{k v} \frac{\partial}{\partial v}
$$

есть симметрия Ли системы

$$
u_{x x}=f(x, u), \quad v_{x}=g(x, u),
$$

связанной с уравнением $u_{x x}=f(x, u)$, то $f$ и $g$ должны иметь вид

$$
g(x, u)=\frac{c(x)}{u^{2}}-\frac{a^{\prime}(x)}{k a(x)}, \quad f(x, u)=d(x) u-\frac{k c^{\prime}(x)}{2 u}-\frac{k^{2} c(x)^{2}}{4 u^{3}} .
$$

\section{3. ЗАКЛЮЧЕНИЕ}

В настоящей работе мы предложили искать нелокальные симметрии ОДУ с помощью погружения этого уравнения во вспомогательную "накрывающую" систему с вспомогательными зависимыми переменными. Поскольку нелокальные симметрии ОДУ являются локальными симметриями соответствующей вспомогательной системы, эта конструкция задает алгоритмический метод поиска нелокальных симметрий такого вида. Это позволяет исследователям использовать компьютер для обычно достаточно трудоемких вычислений.

Нами были найдены некоторые классы ОДУ (9) и (27), для которых не существует симметрий Ли, если не выполнены некоторые специальные условия, но для которых можно найти нелокальные симметрии. С помощью этих нелокальных симметрий порядок этих уравнений можно понизить.

Благодарности. Автор признателен за поддержку со стороны DGICYT (проект MTM2006-05031), Junta de Andalucía group FQM-201 и проекта P06-FQM-01448.

\section{Список литературы}

[1] A. González-López, Phys. Lett. A, 133:4-5 (1988), 190-194.

[2] I. S. Krasil'shchik, A. M. Vinogradov, Acta Appl. Math., 2:1 (1984), 79-96.

[3] I. S. Krasil'shchik, A. M. Vinogradov, Acta Appl. Math., 15:1-2 (1989), 161-209.

[4] А. М. Виноградов, И. С. Красильщик, ДАН СССР, 275:5 (1984), 1044-1049.

[5] А. М. Виноградов, И. С. Красильщик, ДАН СССР, 253:6 (1980), 1289-1293.

[6] G. W. Bluman, G. J. Reid, IMA J. Appl. Math., 40:2 (1988), 87-94. 
[7] M. L. Gandarias, E. Medina, C. Muriel, Nonlinear Anal., 47:8 (2001), 5167-5178.

[8] M. L. Gandarias, E. Medina, C. Muriel, J. Nonlinear Math. Phys., 9, Suppl. 1 (2002), 47-58.

[9] И.Ш. Ахатов, Р. К. Газизов, Н. Х. Ибрагимов, "Нелокальные симметрии. Эвристический подход", Итоги науки и техники. Сер. Соврем. пробл. мат. Нов. достиж., 34, ВИНИТИ, М., 1989, 3-83.

[10] П. Олвер, Приложения групп Ли к дифференииальным уравнениям, Мир, М., 1989.

[11] C. Muriel, J. L. Romero, IMA J. Appl. Math., 66:2 (2001), 111-125.

[12] D. C. Ferraioli, J. Phys. A, 40:21 (2007), 5479-5489.

[13] C. Muriel, J. L. Romero, IMA J. Appl. Math., 72:2 (2007), 191-205.

[14] B. Abraham-Shrauner, K. S. Govinder, P. G. L. Leach, Phys. Lett. A, 203:4 (1995), 169-174.

[15] B. Abraham-Shrauner, IMA J. Appl. Math., 56:3 (1996), 235-252.

[16] B. Abraham-Shrauner, J. Nonlinear Math. Phys., 9, Suppl. 2 (2002), 1-9.

[17] A. A. Adam, F. M. Mahomed, IMA J. Appl. Math., 60:2 (1998), 187-198.

[18] R. M. Edelstein, K. S. Govinder, F. M. Mahomed, J. Phys. A, 34:6 (2001), 1141-1152.

[19] C. Géromini, M. R. Feix, P. G. L. Leach, J. Phys. A, 34:47 (2001), 10109-10117.

[20] N. H. Ibragimov, J. Math. Anal. Appl., 318:2 (2006), 742-757. 